\title{
Sławomir Moździoch, Stanisław Rosik, Thomas Wünsch (dir.), Forschungen zur Geschichte Schlesiens. Methoden und Praxis der Historiographie und neue Untersuchungen
}

Sébastien Rossignol

\section{CpenEdition}

Journals

Édition électronique

URL : http://journals.openedition.org/ifha/8648

DOI : $10.4000 /$ ifha. 8648

ISSN : 2198-8943

Éditeur

IFRA - Institut franco-allemand (sciences historiques et sociales)

Référence électronique

Sébastien Rossignol, « Sławomir Moździoch, Stanisław Rosik, Thomas Wünsch (dir.), Forschungen zur Geschichte Schlesiens. Methoden und Praxis der Historiographie und neue Untersuchungen », Revue de I'IFHA [En ligne], Date de recension, mis en ligne le 01 février 2017, consulté le 24 septembre 2020. URL : http://journals.openedition.org/ifha/8648 ; DOI : https://doi.org/10.4000/ifha.8648

Ce document a été généré automatiquement le 24 septembre 2020.

(C)IFHA 


\title{
Sławomir Moździoch, Stanisław Rosik, Thomas Wünsch (dir.),
} Forschungen zur Geschichte Schlesiens. Methoden und Praxis der Historiographie und neue Untersuchungen

\author{
Sébastien Rossignol
}

\section{RÉFÉRENCE}

Sławomir Moździoch, Stanisław Rosik, Thomas Wünsch (dir.), Silesia historica. Badania nad historiĄ ŚlĄska. Metody i praktyka historiografii oraz nowe poszukiwania. Forschungen zur Geschichte Schlesiens. Methoden und Praxis der Historiographie und neue Untersuchungen, Wrocław: Chronicon (Radices Silesiae - Silesiacae radices, 2), 2012, 146 p., 30 PLN 
Ce volume est le deuxième d'une série dédiée à l'histoire et à l'historiographie de la Silésie. Il comprend des articles rédigés en allemand et en polonais avec, pour chaque contribution, un résumé dans l'autre langue. Les articles comprennent des réflexions sur la méthodologie historique, sur la terminologie utilisée par les historiens et sur les approches historiographiques pour étudier une région dont la recherche historique, en raison de son histoire même, est particulièrement compliquée, aux confins des traditions allemande, polonaise et tchèque.

La première section porte sur des questions méthodologiques et théoriques. Thomas Wünsch ouvre le jeu avec un article consacré au concept de « transgraniczność / transborderness» développé par les chercheurs Zbigniew

Kurcz et Andrzej Sakson pour décrire les formes d'identités régionales se développant dans des zones de contact entre plusieurs cultures. T. Wünsch est convaincu que ce concept peut être utile à l'étude de la Silésie, ayant l'avantage d'insister sur les relations dynamiques sans projeter de jugements de valeur et sans construire une narration téléologique.

Marek Cetwiński analyse le contexte de l'histoire du droit polonais de Gustav Stenzel (1832). Bien que Stenzel ait été de tradition libérale à une époque où une attitude sympathique à la cause polonaise était en vogue, M. Cetwiński démontre qu'il était inspiré par les stéréotypes de son époque sur le rôle civilisateur des Allemands. Marta Młynarska-Kaletynowa explique le contexte de la rédaction de l'Histoire de la Silésie en trois volumes parue dans les années 1930 en Pologne. Comme les travaux existants ne suffisaient pas à présenter un simple état des recherches satisfaisant, le projet a contribué à l'étude fondamentale des sources, et il serait encore utile de le consulter aujourd'hui. Dans un commentaire sur le premier volume de la présente série, Grzegorz Myśliwski souligne l'importance de porter plus d'attention à ce que les sources ont à dire sur les formes d'identités des différents groupes ethniques et religieux qui ont habité en Silésie.

La deuxième section comprend des contributions à l'analyse de la terminologie des sources. Przemysław Siekierka présente les textes des auteurs antiques grecs et romains qui peuvent être mis en lien avec la région actuelle de la Silésie. Il souligne les contradictions des auteurs antiques et de la terminologie utilisée pour décrire les populations du barbaricum, invitant à la prudence concernant des noms de peuplades dont la mention avait une fonction discursive comparable à celle de montagnes et de rivières. Ryszard Kaczmarek s'intéresse aux expressions des identités nationales à propos des officiers et des soldats de Haute-Silésie au XIX ${ }^{e}$ siècle et souligne qu'il est important de lire ces sources en tenant compte du contexte de la modernisation sociale 
et étatique de la période. Jacek DĘbicki exhorte ses collègues à davantage réfléchir aux différences terminologiques des termes utilisés dans les historiographies allemande, polonaise et tchèque et à s'efforcer de s'entendre sur des définitions communes. Ainsi, même des termes apparemment équivalents comme kraj et Land ont des connotations différentes dans leurs langues respectives : kraj est souvent compris comme " peuple", " patrie » en polonais, alors que Land est plutôt « région » en Allemagne, où il est l'objet de la Landesgeschichte.

La troisième section comporte une série d'études de cas. Piotr Boroń attire l'attention sur les problèmes liés à l'étude de la Haute-Silésie aux X ${ }^{\mathrm{e}}$-XII ${ }^{\mathrm{e}}$ siècles. Appelant à une collaboration plus étroite entre historiens et archéologues, il considère qu'il est problématique que les chercheurs transposent souvent à cette période des frontières régionales qui ne sont attestées qu'au Moyen Âge tardif. Dominik Nowakowski résume l'état des recherches sur les changements structurels en lien avec le développement des villes de droit allemand dans la région de Głogów (Glogau) au XIII ${ }^{e}$ siècle. Aleksander Paroń approche le récit de Jan Długosz sur l'invasion mongole de 1241 non pas, comme cela a été généralement le cas dans l'historiographie polonaise, afin de jauger la fiabilité de l'historien $\mathrm{du} \mathrm{XV}^{\mathrm{e}}$ siècle, mais afin de comprendre de quelle façon il s'est servi de l'épisode pour construire une vision particulière de l'histoire polonaise. À l'aide d'une relecture attentive des sources, Stanisław Rosik déconstruit les interprétations traditionnelles des origines du nom de la Silésie, qui aurait son origine dans la peuplade des Sleenzane mentionnée par le « Géographe de Bavière » au IX ${ }^{\mathrm{e}}$ ou X siècle.

Bien que les contributions à ce volume soient éparses et les thèmes discutés assez arbitraires, les questions abordées stimulent une réflexion sur les défis de l'écriture de l'histoire, entre étude des sources, constructions historiographiques et cadres théoriques employés. La Silésie est certes un cas de figure unique qui se prête particulièrement à ce type d'exercice, et pour lequel il est même tout à fait nécessaire, mais les résultats pourront également contribuer à approfondir la réflexion sur les manières d'aborder l'étude d'autres régions historiques. Il reste à espérer que ce volume invitera à une intensification des échanges d'idées et des débats entre chercheurs allemands, polonais et tchèques - voire à entreprendre des études semblables pour d'autres régions au passé particulièrement complexe. Ce livre original témoigne du dynamisme de la recherche polonaise et de son intégration dans le monde académique international ; elle mérite assurément une meilleure reconnaissance.

\section{INDEX}

Thèmes : Histoire des idées, Historiographie/Méthodologie

Index chronologique : Moyen Âge, Époque contemporaine, Ouvrages transpériodiques 


\section{AUTEURS}

\section{SÉBASTIEN ROSSIGNOL}

Memorial University, Terre-Neuve-et-Labrador 